\title{
A New Flexible Tactile Sensor for Contact Position Detection Based on Refraction of Electric Field Lines
}

\author{
Jinhua Ye, Jianfeng Huang, Jianpeng Chen, Haomiao Wang, and Haibin $\mathrm{Wu}^{*}$ \\ School of Mechanical Engineering and Automation, Fuzhou University, \\ 2 Xue Yuan Road, University Town, Fuzhou 350108 China
}

(Received January 9, 2018; accepted July 17, 2018)

Keywords: steady-state current field, potential distribution, flexible tactile sensor, refraction of electric field lines

A new flexible tactile sensor has been designed on the basis of the electric potential distribution of the planar steady current field. The sensor is built to detect information on the contact position for human-robot interaction, such as that involving human-care robots, medical robots, or other service robots. The design scheme of a three-circle conductive plane was proposed according to the refraction of electric field lines by comparing various schemes, which can realize the $X$ and $Y$ coordinate detection of contact positions. An approximately linear relationship between the potential distribution and contact position coordinates was verified through COMSOL simulation, and an error distribution rule for contact position detection on the conductive plane was deduced. Then, a design scheme of the tactile sensor with a three-layer structure was proposed; such a structure consisted of an upper conductive layer, a middle isolation layer, and a lower conductive layer. A mathematical model of contact position detection was established, and the influence of surface contact on detection accuracy was analyzed with COMSOL. A flexible tactile sensor sample was fabricated, and an experiment on contact position detection was conducted on the sensor sample. The feasibility of the sensor was verified with a robot manipulator.

\section{Introduction}

Robot intelligence has received increasing attention from scholars worldwide. ${ }^{(1)}$ The most important objective of robot intelligence research is to make robots acquire senses similar to those of humans and to collect information from the external environment. It also plays an important role in the development of human-robot interaction, ${ }^{(2,3)}$ such as that involving human-care robots, medical robots, or other service robots.

Currently, research on robot environment perception technology mainly focuses on two categories: visual processing and tactile sensing. ${ }^{(4)}$ Visual sensor technology uses cameras to obtain images of the environment and performs image processing to obtain external information. The technology can thus help robots avoid obstacles ${ }^{(5-7)}$ and determine the path 
of motion. ${ }^{(8)}$ For example, Nokata et al. ${ }^{(9)}$ used monocular vision in the manipulator space and calculated the human-robot distance to identify the danger level through model expansion and human body intersection in images. Flacco et al. ${ }^{(10)}$ calculated the human-robot distance to determine danger levels and employed safety control measures in the Kinect depth space. However, the algorithm for vision sensing technology is relatively complex and sometimes not very reliable.

Tactile perception is one of the most important methods for robots to achieve reliable physical interaction with their external environment. With the use of tactile sensors, robots can accurately sense the external environment, make an appropriate response, or employ necessary protective measures.

At present, contact tactile sensors can be grouped into those with an array structure and those with a non-array structure, with the former being dominant. Tactile sensors are used in contact detections, including contact position, contact texture, contact force, and temperature detections. For example, Seminara et al. ${ }^{(1)}$ designed a modular sensor array based on the piezoelectric effect. The module was an equilateral triangle on which a piezoelectric film sensing unit was arranged and supported high sampling frequencies. Büscher et al. ${ }^{(12)}$ introduced a flexible and extensible tactile sensor based on fiber. The sensor used a soft material to cover any natural shape. In addition, the sensor possessed a multilayer structure, in which a force-sensitive resistance layer was sandwiched between two electrode layers. The values measured by the sensor ranged from 1 to $500 \mathrm{kPa}$, which meets the force requirement of human activities. However, due to the array structure of the sensor, the detection of blind spots among tactile sensing units is unavoidable. In addition, large areas of coverage often require the integration of numerous sensors and extensive signal processing, both of which affect the real-time performance and decrease the reliability of the system. ${ }^{(13)}$

The detection area of a non-array tactile sensor is continuous, so its signal processing circuit is simple. Such a sensor is suitable for large areas that cover robot surfaces. Pan and $\mathrm{Zhu}^{(14)}$ proposed a flexible artificial skin that can cover robot surfaces. The skin was designed based on the four-connector technology. First, a voltage was applied to a flexible conductive cloth, and the potential in the contact area of the flexible conductive cloth was detected to determine the contact position. Rana et al. ${ }^{(15)}$ designed a tactile sensor based on capacitance and an improved dielectric material. The sensor was sensitive to an extremely light pressure as well as to a wide range of pressures. However, its response was nonlinear, and it could not detect contact position information. Francesco et al. ${ }^{(16)}$ developed a flexible sensor based on electrical impedance tomography that can locate the area where force was applied. It detected forces applied over a single point on a flat or deformable surface and over multiple points on a flat surface, and changes in the underlying geometry. However, the spatial resolution of this sensor was relatively low. It also could not detect small forces. Zhang et al. ${ }^{(17)}$ introduced Electrick, a lowcost and versatile sensing technique that enables touch input on a wide variety of objects and surfaces, whether small or large, flat or irregular. It also used electrical impedance tomography and could detect multiple points on flat or deformable surfaces. However, the approach relies on the shunting effect, that is, a grounded object, such as a user's finger, will shunt some current to ground when it encounters an electric field through capacitive coupling (similar to surface 
capacitive screens). Thus, the object in contact with the sensor must be capacitive such as a finger or a capacitive stylus. It is not suitable for human-robot collaboration because it ought to detect objects made of any material that come in contact with the robot.

Our team focused on flexible tactile sensors with a non-array structure. In a previous work, ${ }^{(18)}$ a sensor model with a five-layer structure was adopted based on the uniqueness theorem of electric field. With this sensor, a uniform electric field in one direction was generated on one conductive layer, and then a uniform electric field in the other direction was generated on the other conductive layer in the same manner. The position coordinates were determined by detecting the potential value of the contact area on the two conductive layers. This paper presents a three-layer sensor structure model based on existing research. Through the special design of the conductive layer, the sensor can achieve position detection in two directions on the same conductive layer. This design simplifies the sensor structure considerably by making it thin.

\section{Principle of Tactile Sensor}

\subsection{Potential distribution of the planar electric field}

Two electrodes were arranged on a conductive plane with a given conductivity. If the two electrodes add a DC bias voltage, they form a distributed electric field on the conductive plane. The distribution of the electric field on the conductive layer is related to the position of the electrodes and the shape of the conductive plane. As shown in Fig. 1(a), two point electrodes were arranged horizontally on the conductive plane. A DC bias voltage was set. The black line represents the electric field line, and the colored line represents the equipotential line in the electric field. As posited by the potential field theory, the electric potential decreases monotonically along the direction of each electric field line. If the potential value of a point is measured, the approximate position of the point, which is apart from the two electrodes, can be

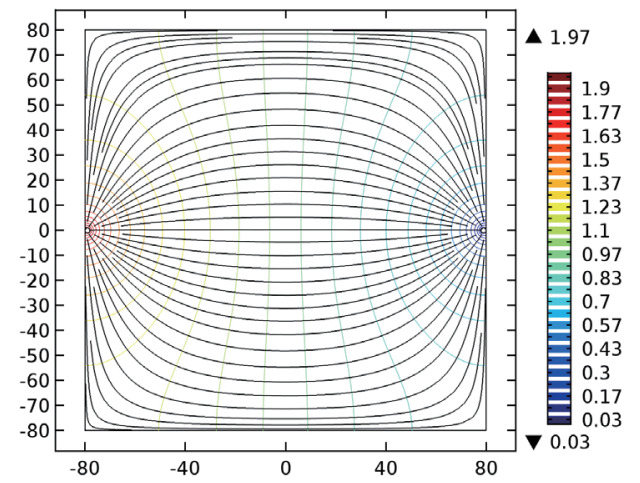

(a)

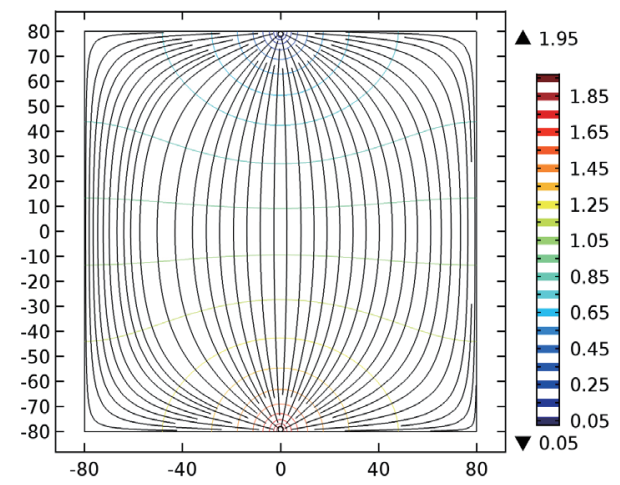

(b)

Fig. 1. (Color online) Electric field distribution of a conductive layer under the action of (a) two horizontal and (b) two vertical point electrodes. 
inferred in the horizontal direction. Similarly, two point electrodes were arranged vertically on the conductive plane, as shown in Fig. 1(b). We can also determine the approximate position in the vertical direction. In fact, the intersection point of the two equipotential lines mentioned above maintained a one-to-one correspondence with the position coordinates of the intersection on the plane. ${ }^{(19,20)}$ By detecting the potential information of a point on the conductive plane, the position coordinates of the point on the plane can be calculated.

The distribution of an electric field is similar to that of an electrostatic field generated by an electric dipole. Therefore, an electric field can be studied on the basis of the electric field theory of electric dipoles. An electrostatic field generated by an electric dipole with a distance of $l$ and a power of $q$ can be used to simulate a planar electric field with a potential difference of $V_{C C}$. The electrostatic field of the electric dipole is calculated using the electric potential superposition method. The electric potential of a point, whose distances from the positive and negative charge points are $r_{1}$ and $r_{2}$, respectively, can be calculated with the following formula: ${ }^{(21)}$

$$
\varphi=\frac{1}{4 \pi \varepsilon_{0}} \frac{q}{r_{1}}+\frac{1}{4 \pi \varepsilon_{0}} \frac{-q}{r_{2}}=\frac{q}{4 \pi \varepsilon_{0}}\left(\frac{1}{r_{1}}-\frac{1}{r_{2}}\right)
$$

where $\varepsilon_{0}$ is the dielectric constant of a vacuum and $\frac{q}{4 \pi \varepsilon_{0}}$ is a constant determined by the DC bias voltage $V_{C C}$.

For an electrostatic field generated by an electric dipole in a vacuum, the zero potential point is on the diagonal bisector $\left(r_{1}=r_{2}\right)$. For a planar electric field, the zero potential point is usually taken at the negative pole of the excitation power supply. Therefore, the potential difference $\varphi^{\prime}$ exists as a result of the selection of the zero potential point in the equivalent process.

According to the symmetry of an electric field, the potential difference is

$$
\varphi^{\prime}=\frac{V_{C C}}{2}
$$

Thus, the potential function of the planar electric field excited by a point electrode is

$$
\varphi=\frac{q}{4 \pi \varepsilon_{0}}\left(\frac{1}{r_{1}}-\frac{1}{r_{2}}\right)+\frac{V_{C C}}{2} .
$$

The coordinate origin is selected to establish a Cartesian coordinate system. The two poles in this coordinate system are $\left(x_{-}, y_{-}\right)$and $\left(x_{+}, y_{+}\right)$, so the potential function is

$$
\varphi=\frac{q}{4 \pi \varepsilon_{0}}\left(\frac{1}{\sqrt{\left(x-x_{-}\right)^{2}+\left(y-y_{-}\right)^{2}}}-\frac{1}{\sqrt{\left(x-x_{+}\right)^{2}+\left(y-y_{+}\right)^{2}}}\right)+\frac{V_{C C}}{2},
$$


where $(x, y)$ are the position coordinates of the measuring point.

Independent potential fields are generated by applying a bias voltage in the horizontal and vertical directions of the conductive plane. Then, the two potential field equations can be calculated to determine the $2 \mathrm{D}$ position coordinates. It can be seen from Eq. (4) that the relationship between the coordinates of the measuring point and the corresponding potential value is nonlinear, so the coordinates $(x, y)$ cannot be easily determined. In order to determine the coordinates easily, the approximately linear relationship between the potential value and the position coordinates should be set. This can be solved by a reasonable design of a conductive layer to form a uniform electric field.

\subsection{Construction of a uniform planar electric field}

\subsubsection{Design of a conductive layer structure based on refraction of electric field lines}

The conductive plane should be a uniform electric field to satisfy the ideal linear relationship between a measuring point potential and its position coordinates. Therefore, the key issue is the construction of a conductive plane that would make the electric field uniform in a certain area on the plane after the bias voltage is applied. For the nonlinear potential distribution in Figs. 1(a) and 1(b), the conductive plane assumes that a uniform and stable conductive layer is formed from the same conductive material. Conversely, if the conductivity of the conductive layer on the conductive plane can be varied, the current density distribution of the planar conductive layer can be made uniform by designing a current flow channel with different impedance characteristics on the plane. In this manner, the effect of approaching a uniform electric field can be achieved. This process requires the conductive layer to exhibit two or more types of media layout according to a given distribution.

When the current flows through the interface between two different media, the two boundary conditions are the continuity of the normal component of the current and that of the tangential component of the electric field strength. These two conditions are observed on both sides of the interface and are generated by the stable current field.

$$
\left\{\begin{array}{l}
j_{a n}=j_{b n} \\
E_{a t}=E_{b t}
\end{array}\right.
$$

The refraction of the electric field lines at the interface is

$$
\frac{\tan \theta_{2}}{\tan \theta_{1}}=\frac{\gamma_{b}}{\gamma_{a}}
$$

The current refraction through two materials with different conductivities is shown in Figs. 2(a)-2(c). When the conductivities of the two media differ significantly $\left(\gamma_{a}>>\gamma_{b}\right)$, the situation at the junction between the good conductor and the poor conductor is shown in Fig. 


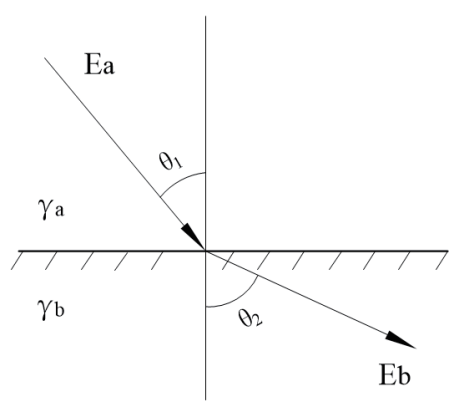

(a)

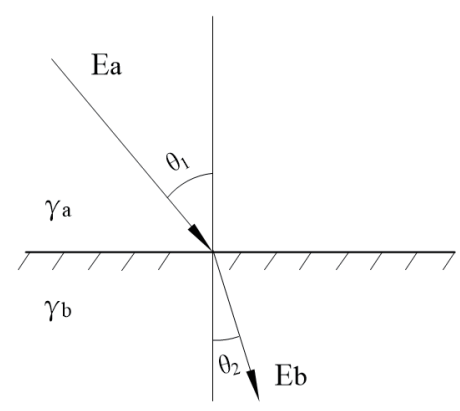

(b)

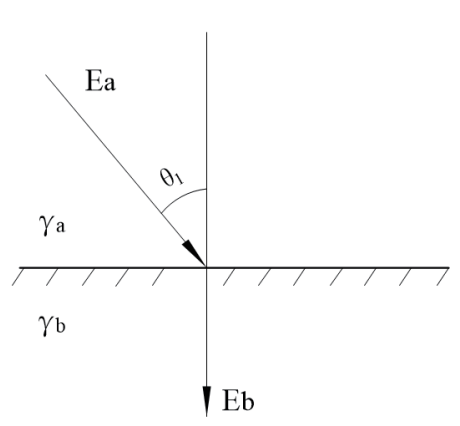

(c)

Fig. 2. Current refraction at an interface. (a) $\gamma_{a}<\gamma_{b}$, (b) $\gamma_{a}>\gamma_{b}$, and (c) $\gamma_{a}>>\gamma_{b}$.

2(c). The refraction angle is approximately equal to zero degrees $\left(\theta_{2} \approx 0^{\circ}\right)$ when the current flows from the region of the medium with high conductivity to the region of the medium with low conductivity, regardless of the incident angle. Therefore, when the conductivity of the material at the junction in the planar electric field differs greatly, the electric field intensity and current density vector at the side of the poor conductor near the interface are approximately perpendicular to the interface, and the side in the poor conductor of the interface can be approximated as an equipotential surface.

Consequently, an electric field distribution that approximates a uniform electric field distribution can be established in the inner rectangular region of the conductive plane by setting the electric conductivities of the different media such that they satisfy the case depicted in Fig. 2(c).

The design of the conductive plane in Fig. 3(a) was then proposed on the basis of the aforementioned setup. The conductive plane was uniformly coated with two different conductive materials, and the region of conductive material $m$ was the region in which a uniform electric field was to be constructed. As long as the electrical conductivity of conductive material $m$ and the electrical conductivity of conductive material $n$ satisfy $\gamma_{n} \gg \gamma_{m}$, a uniform electric field in the $X$-axis direction can be constructed in the detection region. The effective detection area was determined to be a square with a side length of $12 \mathrm{~cm}$ using COMSOL Multiphysics to simulate the structure in Fig. 3(a). As shown in the simulation results presented in Fig. 4(a), an $X$-axis uniform electric field that met the requirements was constructed.

Solving for the coordinates of the measurement point requires two mutually perpendicular potential field equations. Thus, electric fields in the $X$-axis and $Y$-axis directions on the conductive layer must be constructed. According to the construction principle of electric fields in the $X$-axis direction, a conductive area comprising conductive material $n$ was added to the two other sides of the conductive plane ( $Y$-axis direction). The structural design is shown in Fig. 3(b), and the simulation results obtained with COMSOL Multiphysics are shown in Fig. 4(b).

The simulation results indicate that a distortion occurred in the original uniform electric field. This distortion was caused by conductive material $n$ in the $Y$-axis direction disturbing the electric field in the $X$-axis direction. Furthermore, high-conductivity material $n$ on both 


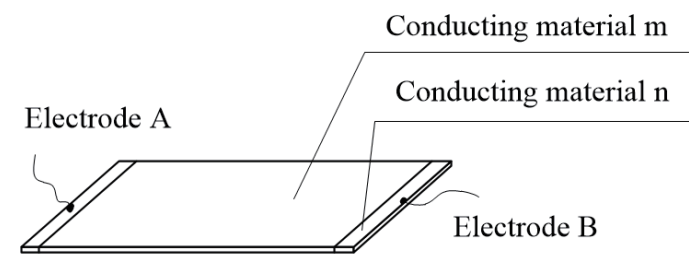

(a)

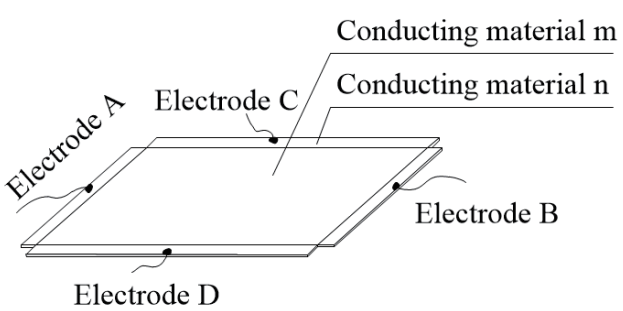

(b)

Fig. 3. (a) Design of a conductive layer in the $X$-axis direction and (b) a two-direction design of the conductive layer.

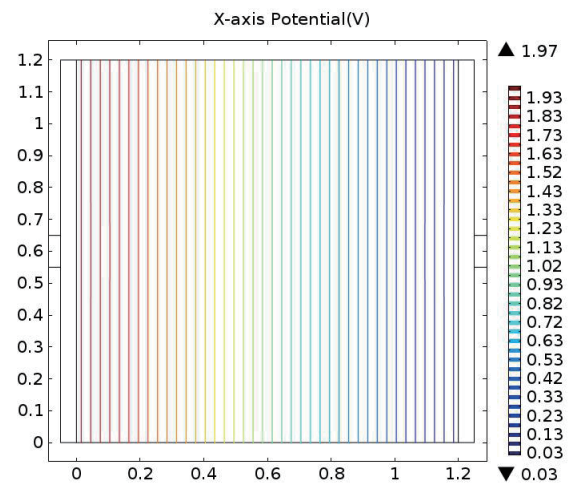

(a)

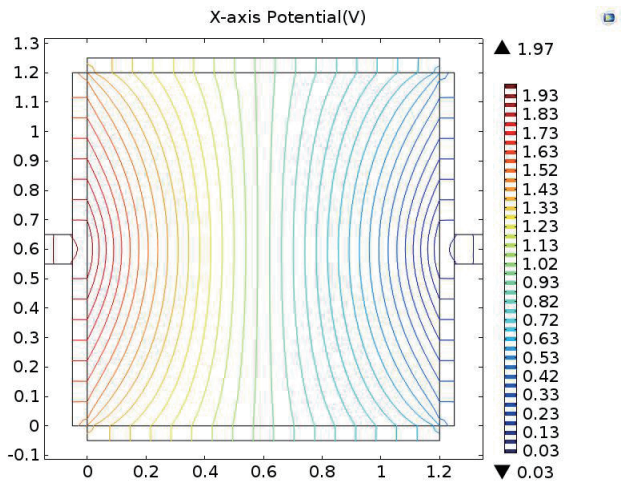

(b)

Fig. 4. (Color online) Potential distributions (a) I and (b) II in the $X$-axis direction.

sides of the $Y$-axis attracted the current in the $X$-axis direction. Therefore, the method of forming a uniform electric field in a single direction of a rectangular plane is simple and easy to implement, but the design of the conductive layer becomes complicated if the goal is to form uniform electric fields separately in two mutually perpendicular directions of the rectangular plane. Figure 2(a) shows that, when $\gamma_{a}<\gamma_{b}$, the refraction current tends to be parallel to the interface for the incident current. Therefore, a pair of materials with a conductivity of $\gamma_{a}<\gamma_{b}$ can be designed based on the previous case to transform the vertical component produced by the original $Y$-axis into an acceptable parallel component.

A series of conductive plane structures were designed and four of them were selected as shown in Fig. 5. Conductive material 1 and conductive material 3 satisfy $\gamma_{1}<<\gamma_{3}$, and they were used to construct the electric field in the detection region. Conductive materials 1 and 2 satisfy $\gamma_{2}<\gamma_{1}$ to correct the influence of the $Y$-axis direction structure when constructing the electric field in the $X$-axis direction. The three conductive materials in four figures were arranged in the same manner, but the connections at the corners differed.

Each structure in Fig. 5 was simulated using COMSOL Multiphysics. The results are shown in Fig. 6. The electric fields constructed with different conductive layer structures differ 


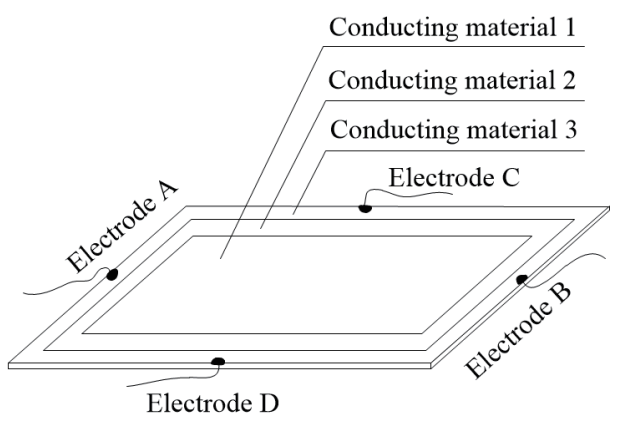

(a)

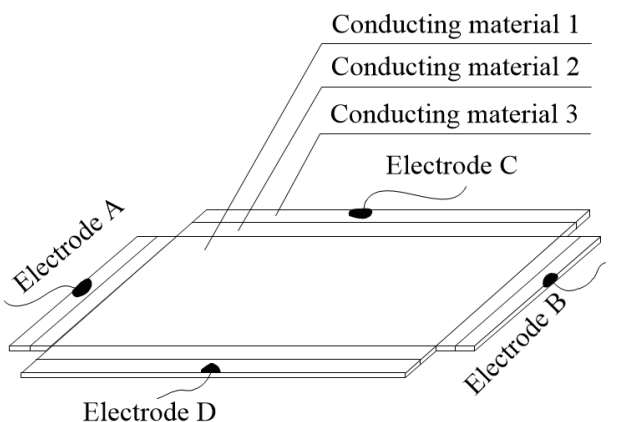

(c)

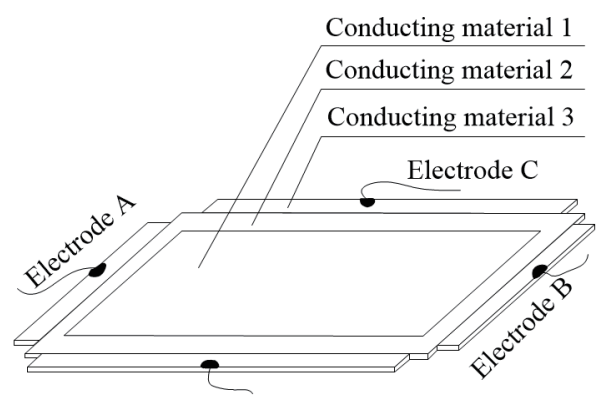

(b)

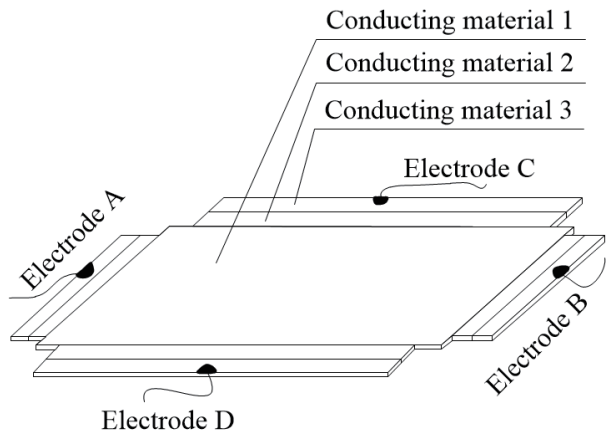

(d)

Fig. 5. Conductive layer structure design (a) 1, (b) 2, (c) 3, and (d) 4 .

significantly. The electric field in Fig. 6(a) is relatively ideal, and the electric fields in Figs. 6(b)-6(d) are considerably different from the uniform electric field. Therefore, we introduced a correlation coefficient to evaluate the different designs for the conductive layer structure.

\subsubsection{Evaluation of conductive layer design using a correlation coefficient}

In the uniform electric field shown in Fig. 4(a), the electric potential in the $X$-axis direction of the conductive layer decreases linearly in relation to the position. A rectangular coordinate system is established at the lower left corner of the detection area. On line $y=n(n<$ side length, $n$ is a constant), potential $V$ is linearly related to position $x$, and the relationship is fixed and unique. Therefore, we select three representative lines in the detection area, namely, $1, y=0.10$ $\mathrm{dm} ; 2, y=0.40 \mathrm{dm}$; and 3, $y=0.60 \mathrm{dm}$ [Fig. 7(a)]. The trend of the potential variation with the position on the three lines is shown in Fig. 7(b). As a result of the linear and unique relationship between the electric potential and its position in the uniform electric field, the three trend lines in Fig. 7(b) are coincident.

Correlation coefficients are statistical indexes used to reflect the degree of correlation between variables. A correlation coefficient is calculated as

$$
r_{x v}=\frac{\operatorname{Cov}(x, v)}{\sqrt{D(x)} \sqrt{D(v)}}
$$




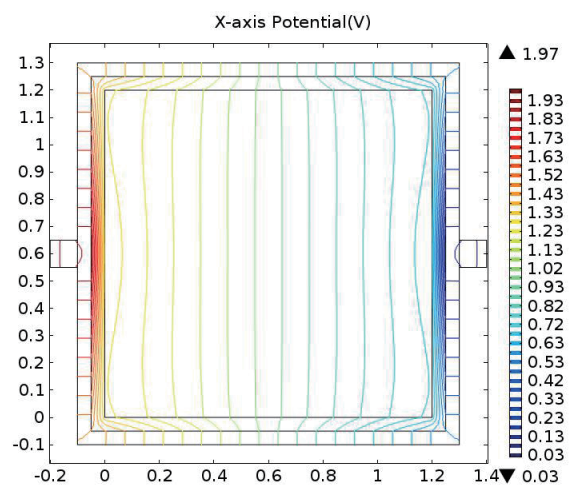

(a)

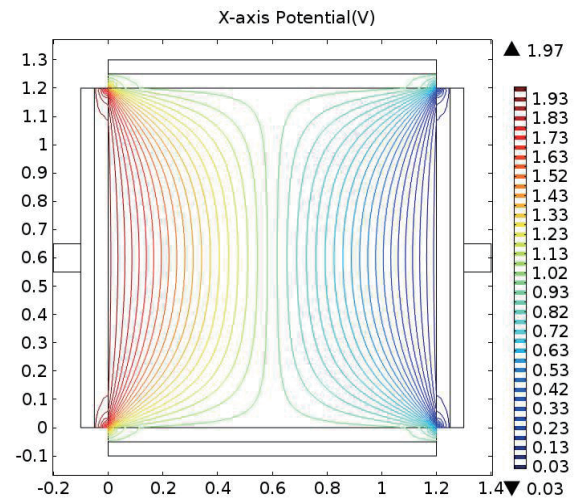

(c)

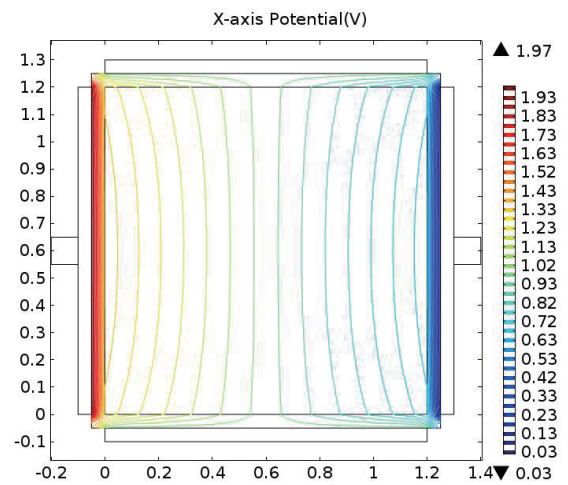

(b)

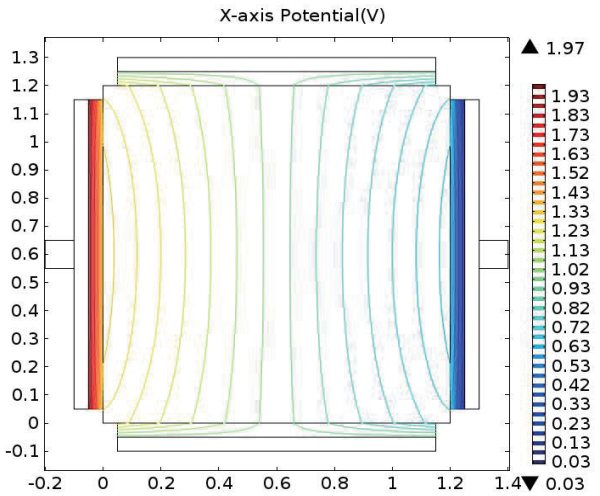

(d)

Fig. 6. (Color online) Simulation results of conductive layer structure design. (a) 1, (b) 2, (c) 3, and (d) 4 .

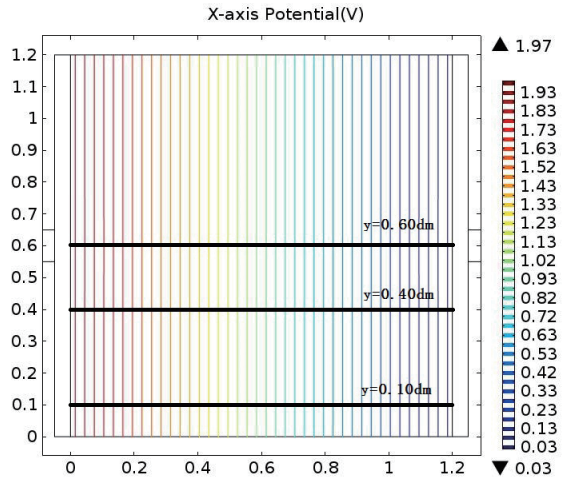

(a)

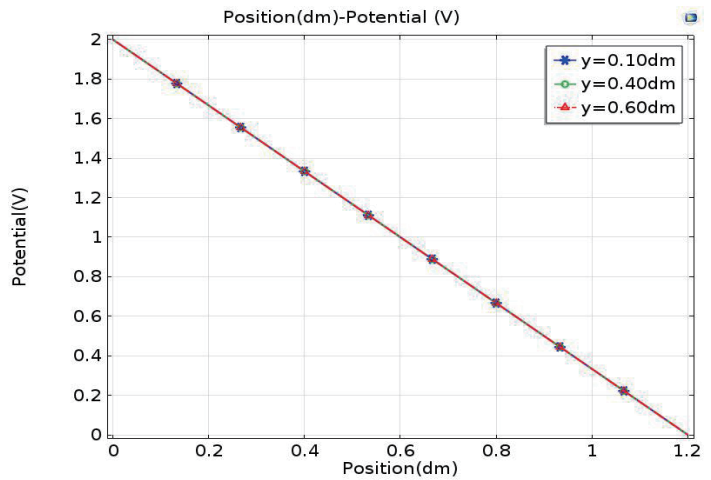

(b)

Fig. 7. (Color online) (a) Selection of the relationship between potential and position in the detection area. (b) Trends of potential and position in the uniform electric field.

where $\operatorname{Cov}(x, v)$ is the covariance of $x$ and $v . D(x)$ and $D(v)$ are the variances of $x$ and $v$, respectively.

The correlation coefficient is between -1 and $+1(-1 \leq r \leq+1)$. When $r>0$, the two variables are positively related. When $r<0$, the two variables show a negative correlation. When $|r|=1$, 
the two variables show a completely linear correlation. When $r=0$, no linear correlation exists between the two variables.

The correlation coefficient for the data in Fig. 7(b) for the potential and position of a uniform electric field is -1 . This value indicates that the electric potential and position in the uniform electric field are completely linearly related and that the structure shown in Fig. 7(a) depicts a completely negative correlation.

The correlation coefficient of each design scheme in Fig. 5 was calculated with the same method to determine performance.

First, we derived the relationship between the potential $V$ and position $x$ on $y=0.10 \mathrm{dm}, y=0.40$ $\mathrm{dm}$, and $y=0.60 \mathrm{dm}$ as shown in Fig. 8. The dispersion relationship between the potential and position in Fig. 8(c) is the most severe. The dispersion relationship between the potential and position in Figs. 8(b) and 8(d) is relatively serious. The relationship between the potential and position in Fig. 8(a) is the most concentrated. These results are consistent with the simulation results in Fig. 6.

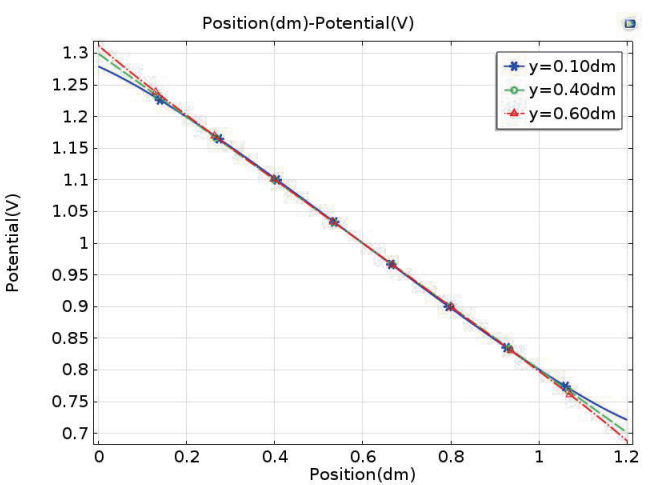

(a)

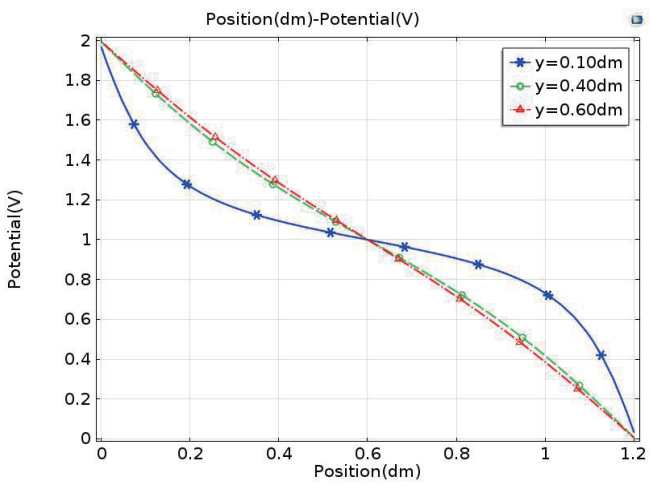

(c)

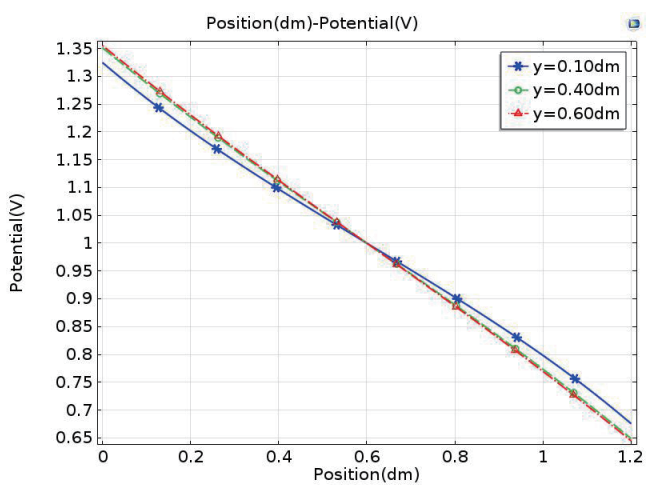

(b)

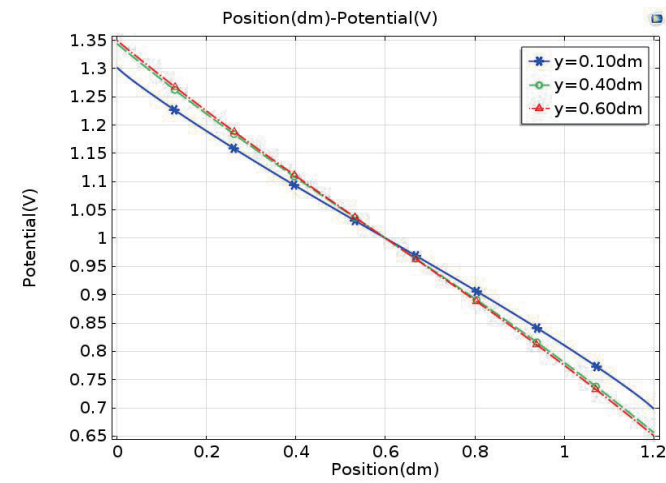

(d)

Fig. 8. (Color online) Relationship between the potential and the position for conductive layer structure schemes (a) 1 , (b) 2, (c) 3, and (d) 4 . 
We then calculated the correlation coefficients of the potentials and positions for four conductive layer design schemes, as shown in Table 1.

Table 1 indicates that $\left|r_{1}\right|>\left|r_{2}\right|>\left|r_{4}\right|>\left|r_{3}\right|$. Thus, the conductive layer design in Fig. 5(a) is the best. In addition, because $|r|=0.999664909 \approx 1$, design 1 is close to the full linear correlation of the uniform electric field.

\subsubsection{Determination of the conductive layer structure design scheme}

The $X$-axis and $Y$-axis electric fields were simulated with COMSOL Multiphysics. The results are shown in Figs. 9(a) and 9(b). The electric field in the $X$ - and $Y$-axes of the conductive layer can be constructed ideally with this design scheme according to the figure. The enlarged region in Fig. 9(a) is a schematic of the direction of the electric field lines on the conductive plane. In the circular enlargement region, regardless of the angle of incidence $\theta_{1}, \theta_{2} \approx 0^{\circ}$ and $\theta_{3}$ $\approx 0^{\circ}$ at the second interface. Hence, the horizontal component can form a uniform electric field in the detection region. In the elliptical enlargement region, regardless of $\theta_{2}{ }^{\prime}, \theta_{3}{ }^{\prime} \approx 90^{\circ}$. Thus, the vertical component affecting the detection area can be effectively eliminated.

In summary, electrically conductive material 1 is a region to be constructed with a uniform electric field, that is, a region for position coordinate detection. By applying a given voltage across the $X$ - and $Y$-axes time-divisionally, a uniform electric field in two directions can be generated on a single conductive plane. The position information of a point on the plane can be obtained by measuring the potential value of a point, that is, the $X$ and $Y$ coordinates.

Table 1

Correlation coefficients for different design schemes.

\begin{tabular}{lrrrr}
\hline Design program serial number & 1 & 2 & 3 & 4 \\
\hline Correlation coefficient & -0.999664909 & -0.998352041 & -0.966078387 & -0.997101704 \\
Absolute value of correlation coefficient & 0.999664909 & 0.998352041 & 0.966078387 & 0.997101704 \\
\hline
\end{tabular}

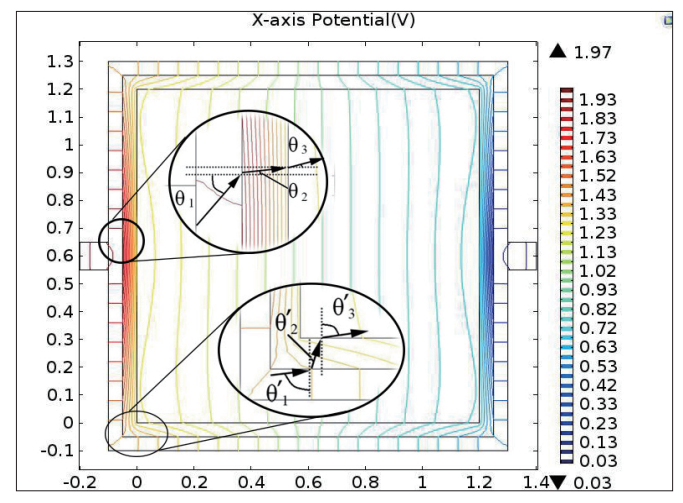

(a)

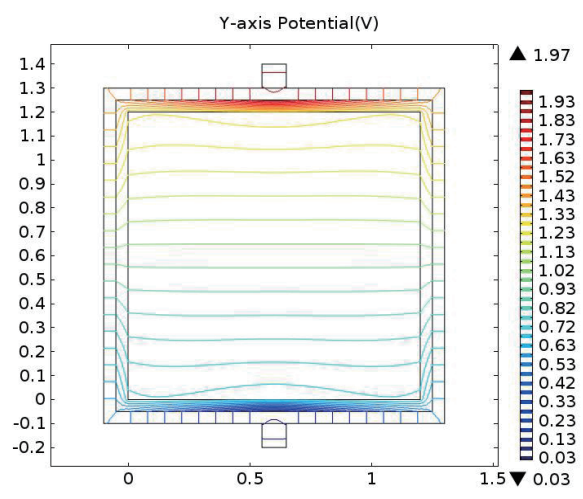

(b)

Fig. 9. (Color online) Potential distribution of the conductive plane of the sensor in the (a) $X$ - and (b) $Y$-axes. 


\section{Tactile Sensor Structure}

\subsection{Structural design of the tactile sensor}

Based on the constant electric field, a uniform electric field can be realized in a certain area by controlling the distribution of current density. The current density distribution can be controlled by coating materials of different conductivity on the rectangular plane according to the law of refraction of current at an interface. Therefore, a three-layer sensor structure (Fig. 10) was further proposed. From top to bottom, the three layers are an upper conductive layer, an intermediate spacer layer, and a lower conductive layer. The upper conductive layer is formed by attaching a uniform conductive dielectric surface on one surface of the film substrate. This layer contains a measurement lead wire. The lower conductive layer is the conductive plane composed of three conductive materials with different resistance characteristics, and it contains electrodes A, B, C, and D. The lower conductive layer is also attached to a film substrate material. The film substrate material exhibits good flexibility and elasticity, and it can be attached to any external surface of a robot or manipulator. This material can also reduce the impact force and protect the conductive material under external force. The isolation layer is a flexible insulated layer with mesh that separates the upper conductive and lower conductive layers in the absence of external force. A pair of electrodes (A and B or C and D) of the lower conductive layer is connected to the positive and negative electrodes of the DC power supply, and an electric field similar to the uniform electric field is formed in the detection region of the lower conductive plane.

By applying a bias voltage to the two sets of electrodes time-divisionally, two uniform electric fields in different directions are generated on the conductive layer time-divisionally to measure the 2D position coordinates. As shown in the structure in Fig. 10, the tactile sensor based on the planar electric field generates two independently distributed electric fields using the lower conductive layer. This tactile sensor obtains position information by establishing mathematical models of potential and position.

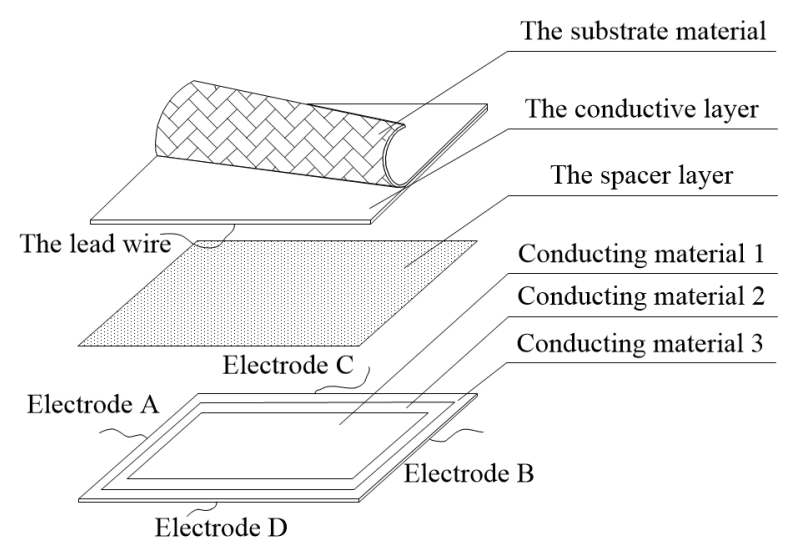

Fig. 10. Structural model of the tactile sensor. 


\subsection{Position detection model of tactile sensor}

\subsubsection{Principle of position coordinate detection in the $X$-axis}

Electrodes A and B of the lower conductive layer are connected to the positive and negative electrodes of the DC power source, respectively. A uniform electric field in the $X$-axis is formed in the detection region (signed by the red line in Fig. 11), and Cartesian coordinate system xoy is established in the detection region. In Fig. 11(a), the line with arrows is the electric field line, and the line perpendicular to the electric field line is the equipotential line. The potential of the $X$-axis in the detection region is computed as

$$
\varphi_{x}=E x=\frac{V_{C C}^{\prime}}{l_{X}} x
$$

and the formula conversion is

$$
x=\frac{\varphi_{x}}{V_{C C}^{\prime}} l_{X}
$$

where $E x$ is the electric field strength, $\varphi_{x}$ is the measured potential of the contact point, $l_{X}$ is the effective width of the detection region, and $V_{C C}^{\prime}$ is the voltage drop in the detection region.

\subsubsection{Principle of position coordinate detection in the $\boldsymbol{Y}$-axis}

Electrodes $\mathrm{C}$ and $\mathrm{D}$ of the lower conductive layer are connected to the positive and negative electrodes of the DC power source, respectively. Then, a uniform electric field in the $Y$-axis is formed in the detection region, and Cartesian coordinate system xoy is established in the detection region, as shown in Fig. 11(b). Similarly to that of the $X$-axis, the potential of the $Y$-axis in the detection region is computed as

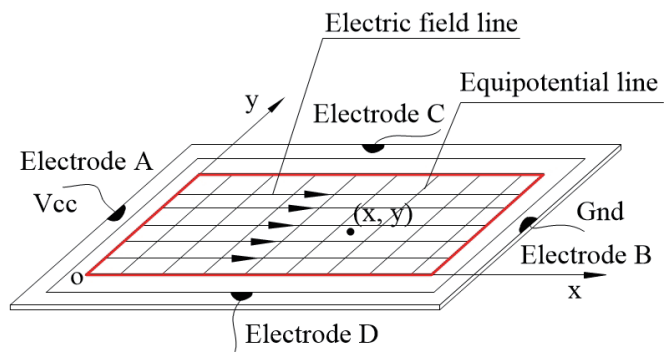

(a)

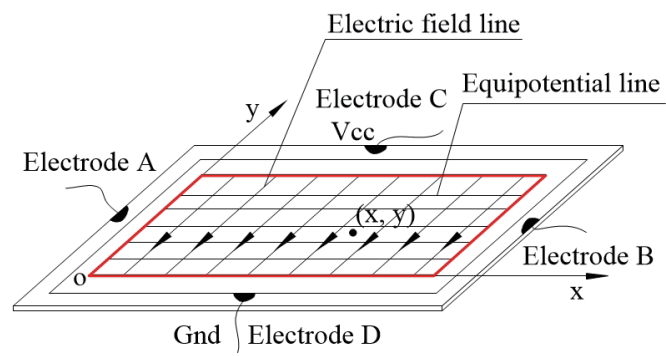

(b)

Fig. 11. (Color online) Schematic of potential detection in the (a) $X$ - and (b) $Y$-axes. 


$$
\varphi_{y}=E y=\frac{V_{C C}^{\prime}}{l_{Y}} y
$$

and the formula conversion is

$$
y=\frac{\varphi_{y}}{V_{C C}^{\prime}} l_{Y}
$$

where $E y$ is the electric field strength, $\varphi_{y}$ is the measured potential of the contact point, $l_{Y}$ is the effective length of the region, and $V_{C C}^{\prime}$ is the voltage drop in the detection region.

\subsubsection{Solution of the electric field}

The coordinates $(x, y)$ of the contact point can be obtained simultaneously with Eqs. (9) and (11). The aim of the entire process of measurement is to find the equipotential lines of the potentials of the two sets of electric fields, as well as their intersection points, as shown in Fig. 12(a).

\section{Simulation}

\subsection{Establishing the sensor model}

With the sensor layer structure shown in Fig. 10, a 3D simulation model [Fig. 12(b)] was established. The upper conductive layer was selected from a material with excellent electrical conductivity, which serves as the measurement lead layer, and it was sectioned to view the lower structure clearly. The small cylinder in the figure is in contact with the upper and lower

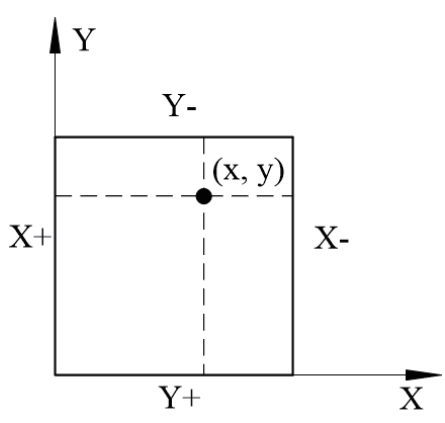

(a)

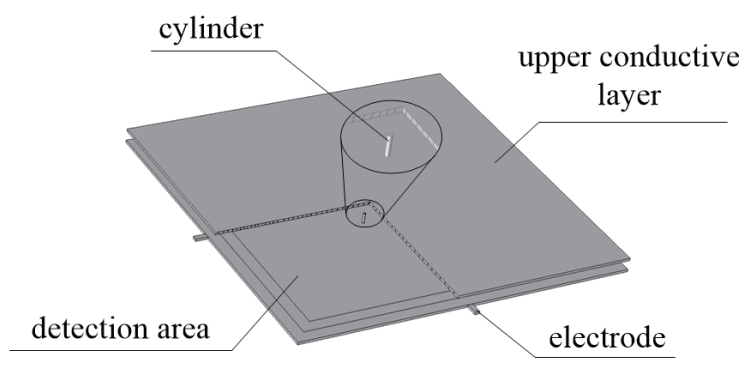

(b)

Fig. 12. (a) Schematic of electric field position detection. (b) 3D simulation model of the sensor. 
conductive layers. This cylinder was used to simulate the contact area. Point and surface contacts, which may exist in reality can be modeled by varying the radius of the cylinder. The shape of the lower conductive layer is a square with a side length of $14 \mathrm{~cm}$ and a thickness of $0.1 \mathrm{~cm}$. The side length of the square detection area is $12 \mathrm{~cm}$. The widths of the two outer rectangular areas are $0.5 \mathrm{~cm}$, and the resistivity ratio of the three square areas from outside to inside is 1:100:10. The structures used in this work were symmetrical in the $X$-axis and $Y$-axis directions, so the measurement processes in the $X$-axis and $Y$-axis directions were exactly the same. Hence, we only discuss the error distribution of measuring the $X$-coordinate in the simulation.

\subsection{Simulation of point contact}

The point contact was simulated by applying a DC voltage of $2 \mathrm{~V}$ to the left electrode in the $X$-axis direction, grounding the right electrode, and setting the radius of the small cylinder in the contact area to $r=0.5 \mathrm{~mm}$. The relationship between the position error and its coordinates was determined through a simulation. In the simulation, we selected a series of points in the detection range of the conductive plane. We analyzed the point contact errors of several points, which were on straight lines $y=10 \mathrm{~mm}, y=40 \mathrm{~mm}$, and $y=60 \mathrm{~mm}$. The relationship between the position error and the $X$-coordinate is shown in Fig. 13. As shown in Fig. 13, the position error of the simulation is not more than $2 \mathrm{~mm}$ in the case of the point contact. The position error distribution is symmetric with respect to the center line. The error decreases as the position approaches the middle area, and the position error increases from the intermediate position toward the two electrodes. The point on straight line $y=10$ is larger than those of the two other lines because the electric field is distorted by the edge effect. Moreover, the position error increases and the electric field is distorted when closer to the boundary.

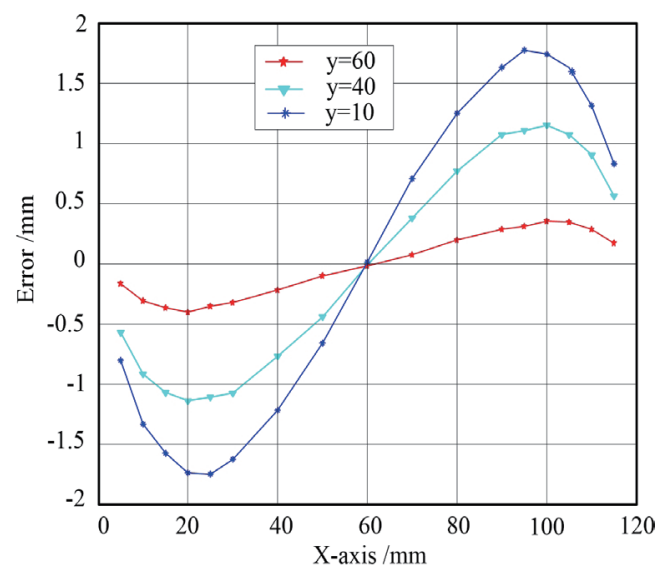

Fig. 13. (Color online) Results of simulation of sensor point contact. 


\subsection{Simulation of surface contact}

The radius of the cylinder was set to $r=12 \mathrm{~mm}$ to simulate surface contact, and the potential distribution under surface contact is shown in Fig. 14(a). We defined the position error as the difference between the measured value and the center coordinate of the cylinder. The results are shown in Fig. 14(b). The figure shows that the surface contact error is slightly larger than the point contact error, but its error distribution trend coincides with the error distribution trend of point contact. The maximum detection error of surface contact is less than $6.0 \mathrm{~mm}$, which is less than the radius of the contact area $(r=12 \mathrm{~mm})$. This result indicates that the detected coordinate value is still within the contact area.

To observe the position error, we continued to analyze the error distribution of the equipotential line of the electric field. In the uniform electric field, the equipotential lines in the $X$-axis direction are parallel to the $Y$-axis. We measured the maximum and minimum potential values on the line parallel to the $Y$-axis $\left(0<x_{i}<120\right)$ and compared them with the potential values on the $x=60 \mathrm{~mm}$ line to obtain the error distribution of the equipotential line and the position error distribution of the sensor [Figs. 15(a) and 15(b), respectively]. The maximum and minimum potential values on the line are the parameters that determine the contact position error. The maximum potential in a series of lines is basically at the boundary, and the minimum potential appears in the middle. Figure 15(b) shows that the error distribution of the sensor satisfies that the error in the middle area is relatively small and the errors at the two ends are relatively large. However, the position error of any point in the detection area of the sensor does not exceed $6 \mathrm{~mm}$.

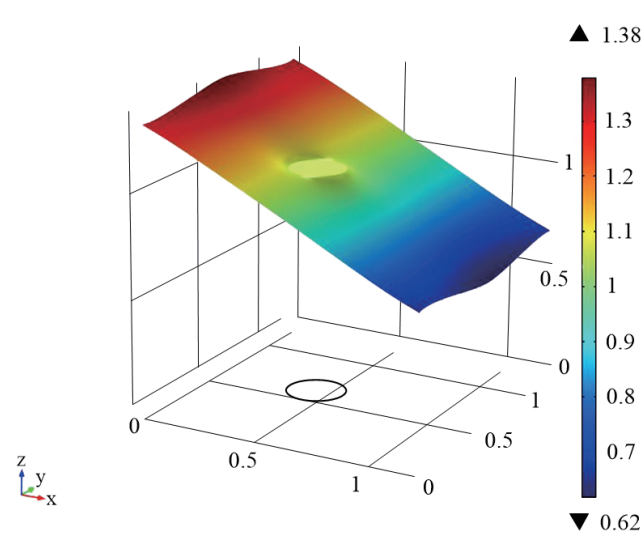

(a)

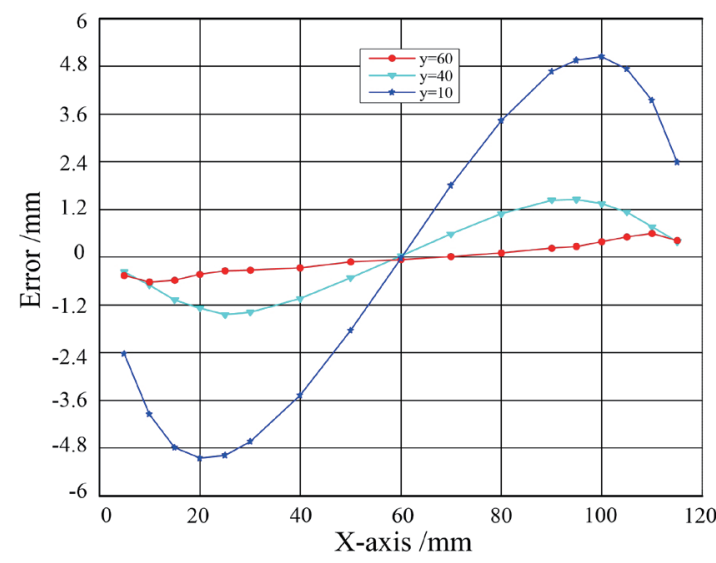

(b)

Fig. 14. (Color online) (a) Potential distribution of surface contact. (b) Results of simulation of sensor surface contact. 


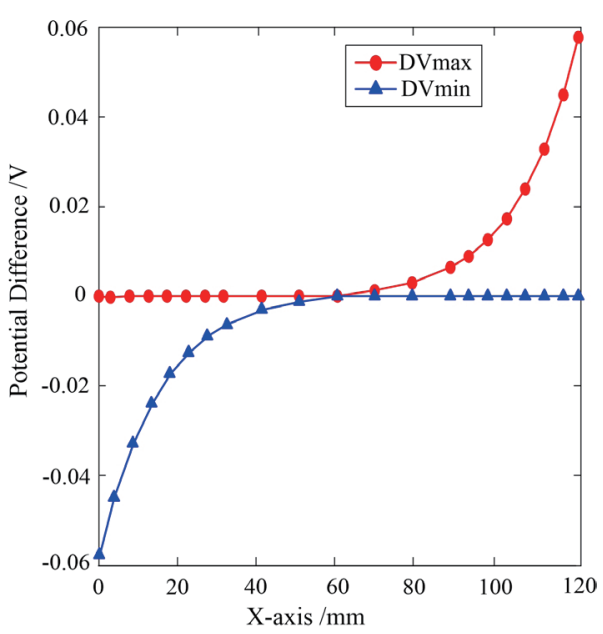

(a)

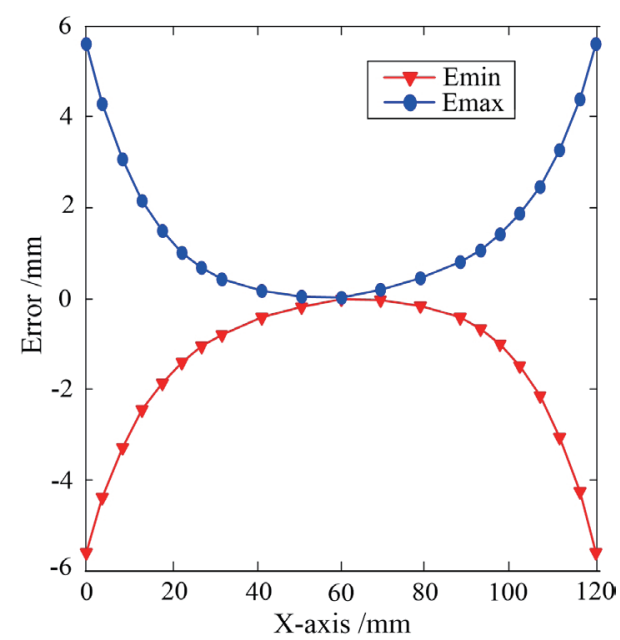

(b)

Fig. 15. (Color online) (a) Error distribution of equipotential line in the $X$-axis direction. (b) Distribution of error in the $X$-axis direction.

\section{Sensor Fabrication and Experimentation}

\subsection{Fabrication of sensor samples}

The effectiveness of the design of the proposed sensor depends on whether it can produce the lower conductive layer. The nature of the lower conductive layer material directly determines the success or failure of the position detection. To meet the material conductivity requirements of the lower conductive layer, we used a customized indium-tin oxide (ITO) conductive film as the conductive layer material and aluminum foil as the electrode material. Since the ITO film is flexible, our sensor could be more flexible. However, according to the property of the ITO film, our sensor has the limit of the bend radius. Grego et al. ${ }^{(22)}$ researched the relationship between the resistance and bend radius of the ITO film. We concluded from his paper that the change in resistance is almost zero when the bend radius does not exceed a certain value. We determined that the minimum bend radius of our ITO film is $4.32 \mathrm{~mm}$. The fabrication of a square conductive layer with a side length of $14 \mathrm{~cm}$ proceeded as follows.

1. The ITO conductive film whose resistivity is $1 \times 10^{-3} \Omega \cdot \mathrm{cm}$ was cut to a square with a side length of $14 \mathrm{~cm}$. A square with a side length of $12 \mathrm{~cm}$ in the middle part of the film was removed, only leaving a frame.

2. Two rectangular sheets of aluminum foil with a length of $14 \mathrm{~cm}$ and a width of $0.5 \mathrm{~cm}$, and the other two with a length of $13 \mathrm{~cm}$ and a width of $0.5 \mathrm{~cm}$ were cut, then attached to the four outer boundaries of the frame mentioned in step one.

3. The ITO conductive film whose resistivity is $1 \times 10^{-4} \Omega \cdot \mathrm{cm}$ was cut to a square with a side length of $12 \mathrm{~cm}$. This film was attached to the center of the frame. 
4. The conductive tape was attached to the junction between the aluminum foil and the ITO conductive film and the junction between the two ITO conductive films. The lower conductive layer sample is shown in Fig. 16(a).

5. The mesh-type insulation layer was made of insulating polyethylene. A square aluminum foil with a side length of $14 \mathrm{~cm}$ was attached to foamed silica gel as the upper conductive layer.

6. The upper conductive, intermediate spacer, and lower conductive layers were arranged in the pattern shown in Fig. 10. The sample sensor was then complete, as shown in Fig. 16(b).

\subsection{Experiments on the tactile sensor}

\subsubsection{Experiments on point contact}

An experiment was conducted using the sensor sample shown in Fig. 16(b). Considering symmetry, we only experimentally validated the position information in the $X$-axis direction. The positive and negative electrodes of the DC bias voltage were applied to the electrodes in the $X$-axis direction. To compare the experimental results with the simulation results, we selected a series of points on lines $y=10 \mathrm{~mm}, y=40 \mathrm{~mm}$, and $y=60 \mathrm{~mm}$ in the detection range of the conductive layer. A voltmeter probe was directly pressed on the given position (regarded as point contact) to read the voltage, and then we converted the measured voltage into the position coordinates according to Eq. (9). The position coordinates obtained were then compared with the actual position coordinates. The experimental results are shown in Fig. 17(a), and the relationship between the position error and the position coordinates is shown in Fig. 17(b). The distribution trend of the position error is consistent with that in the simulation, but the precision of detection is decreased. The error distribution is still symmetrical, and the detection accuracy of the middle region is relatively high. Furthermore, the maximum position error within the detection area does not exceed $3 \mathrm{~mm}$. The increase in error was caused by two

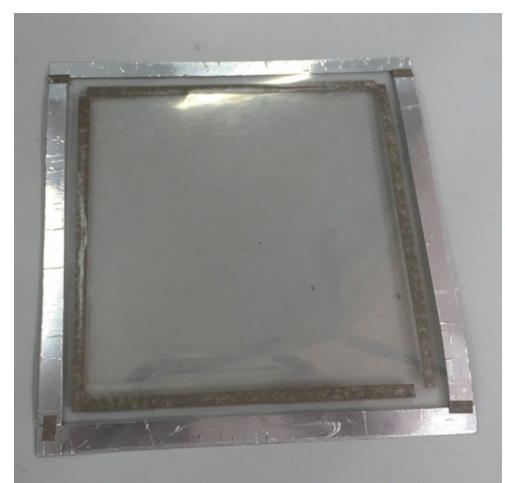

(a)

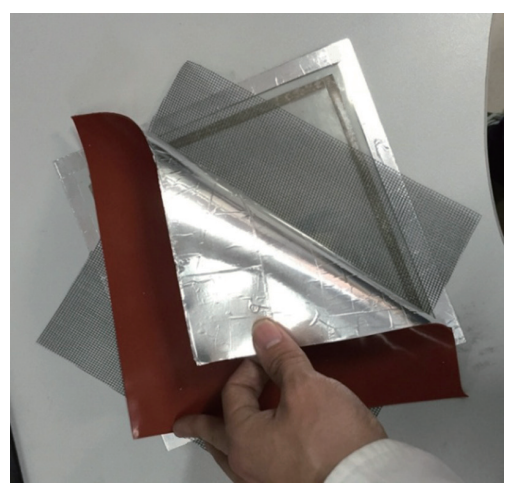

(b)

Fig. 16. (Color online) (a) Sample of the lower conductive layer. (b) Sensor sample. 


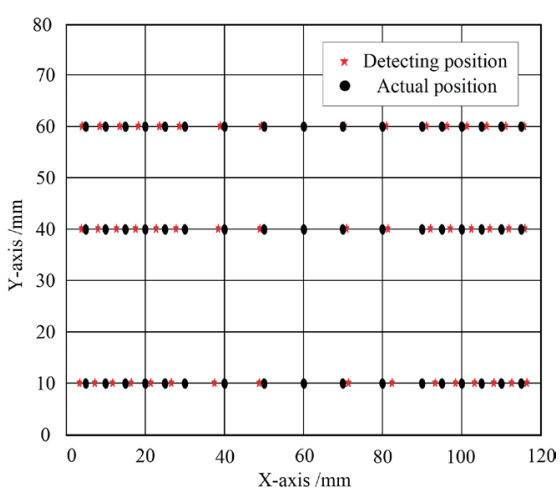

(a)

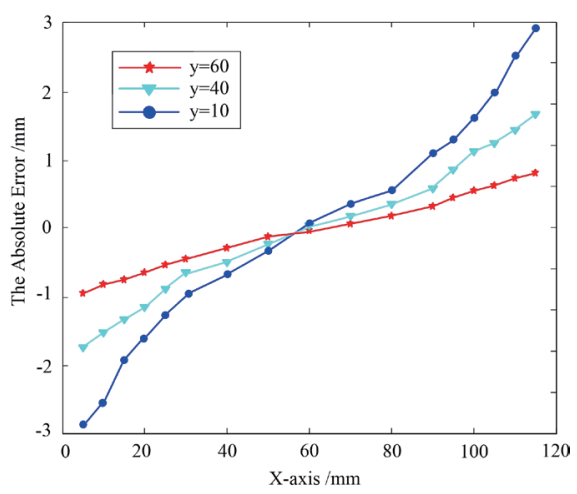

(b)

Fig. 17. (Color online) (a) Results of the point contact experiments. (b) Location error distribution in the point contact experiment.

factors: (1) the properties of the conductive materials, that is, the ITO conductive materials, were not sufficiently uniform and (2) the process of creating the sensor was relatively crude, and deviations existed in the geometry of the cut materials. Although the detection accuracy needs improvement, the trend diagram for the error distribution indicates that the sensor can still meet the requirements of the application as long as appropriate corrections are made at certain specific locations.

\subsubsection{Experiments on surface contact}

Experiments on surface contact were also conducted using the sensor shown in Fig. 16(b). A dsPIC30F3012 single-chip microcomputer system was used to control the switching of the two pairs of voltage circuits of the sensor and achieve data acquisition, amplification, and analogto-digital conversion functions. A ZQ-21B-4 small pressure test machine was used to apply pressure. The entire device is shown in Fig. 18.

The experiment was conducted with the radius $r=9.5 \mathrm{~mm}$ (size of a coin) as the size of the surface contact. To compare the experimental results with the simulation results, we selected a series of points on straight lines $y=10 \mathrm{~mm}, y=40 \mathrm{~mm}$, and $y=60 \mathrm{~mm}$ in the detection range of the sensor. The experimental results are shown in Fig. 19(a), and the results of the measurement errors (the difference between the measured values and the center coordinates of the coin) and position coordinates are shown in Fig. 19(b). The distribution tendency of the surface contact position error coincides with that of the point contact. For surface contact, the most important factor is not the magnitude of the measured position error but whether the position of the measured value lies within the contact area. According to the experimental results, the peak position error in surface contact does not exceed $6 \mathrm{~mm}$, which is less than the radius of the contact area $(r=9.5 \mathrm{~mm})$. This outcome indicates that the measured position is located within the contact area and that the sensor can work effectively. 


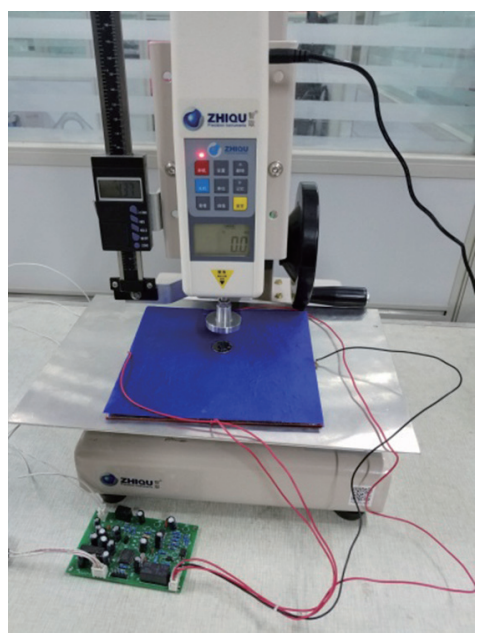

Fig. 18. (Color online) Experimental setup.

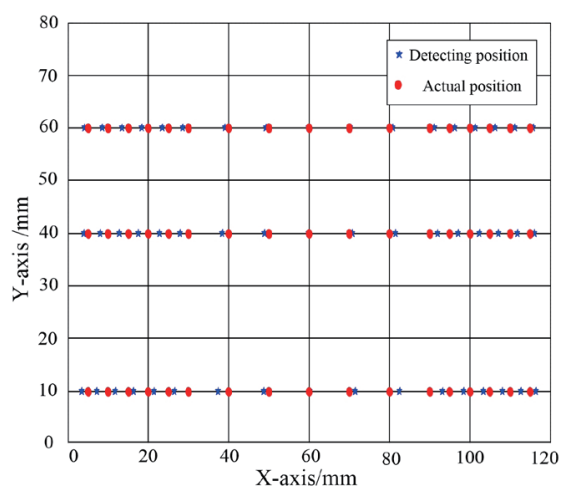

(a)

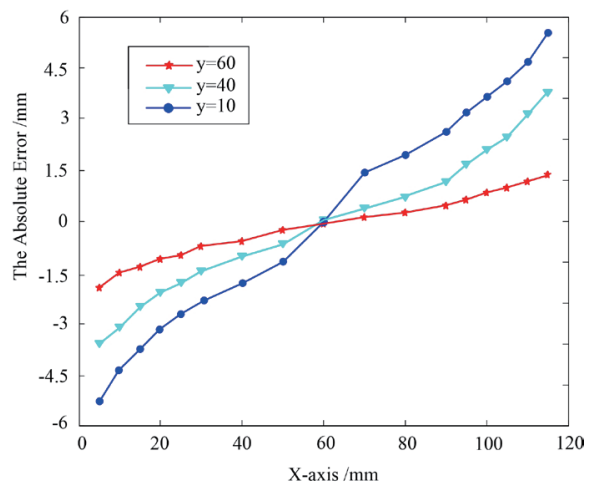

(b)

Fig. 19. (Color online) (a) Results of the surface contact experiment. (b) Location error distribution in the surface contact experiment.

\subsubsection{Surface contact under bending status}

To test the precision of the sensor under the bending status, the industrial robot UR5 with six degrees of freedom was used, and its third link was utilized as an example. Considering the size of the manipulator arm, we fabricated a new sensor whose effective detection region is a square with a side length of $212 \mathrm{~mm}$, then attached it to the third link of the UR5 manipulator, as shown in Fig. 20(a).

Because the error is larger under surface contact according to the results of the above analysis, we just need to analyze the surface contact to simulate the worst situation. A rubber 


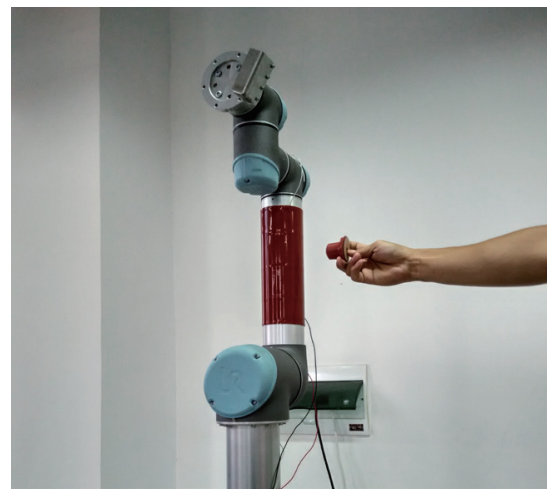

(a)

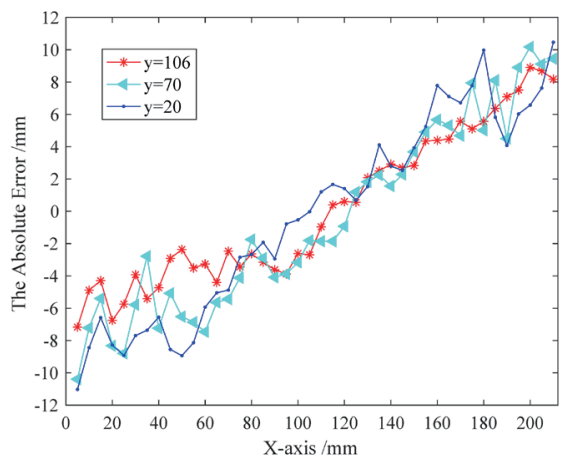

(b)

Fig. 20. (Color online) (a) Setup of measuring bending effect. (b) Location error distribution in the surface contact under bending status.

button with the radius $r=15 \mathrm{~mm}$ was used to press the given area. The error is expressed in the difference between the measured values and the center coordinates of the button. Similar to the distribution of the measured points analyzed above, a series of points on straight lines $y=$ $20 \mathrm{~mm}, y=70 \mathrm{~mm}$, and $y=106 \mathrm{~mm}$ on the sensor of this experiment are selected. The errors on these points are shown in Fig. 20(b). It can be seen that the distribution tendency under the bending status coincides with that under the plane status. The fluctuation of the data is obvious and the smoothness of the data curves is not well compared with that mentioned above. This is because the bending affected the electric field distribution and manually placing rubber button led to the positional errors. However, the measured values still lie within the contact area.

\subsection{Robot collision experiment}

In this experiment, the results measured in Sect. 5.2.3 are used to control the UR5 robot. When collision occurred on one side of the robot in the detection region, the robot would move to another side. The sensor detects the contact coordinates and sends them to the control system via the processing circuit. The control system converts the coordinate system of the sensor into that of the robot. Then, an instruction that is away from the collision position is sent to the joint driver. The experimental process is shown in Fig. 21. When collision occurs, the tactile sensor can detect the collision signal and transmit it to the control system in real time so that the control system can promptly implement an effective collision control strategy. The experimental results show that the tactile sensor designed in this work can be wrapped in the robot arm and respond in time when collision occurs. Hence, it improves the security of human-robot interaction and effectively meets the requirements of practical applications. 


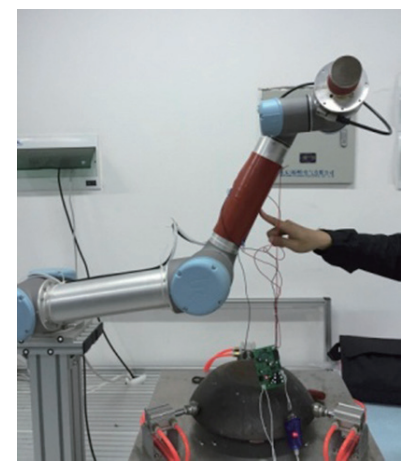

(a)

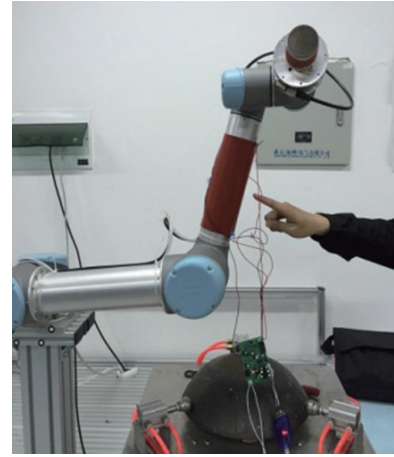

(c)

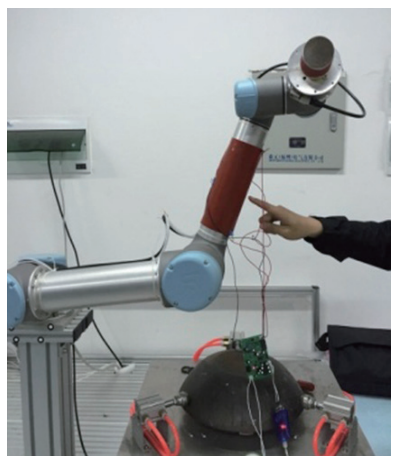

(b)

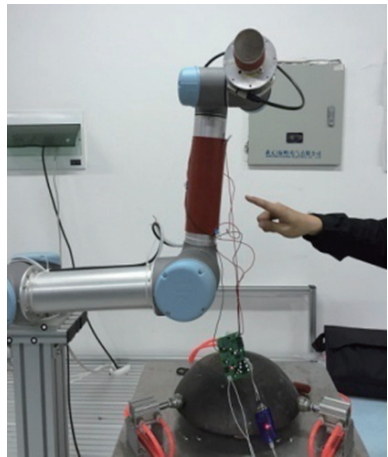

(d)

Fig. 21. (Color online) Experimental process of contact. (a) External force is applied to the robot arm. (b)-(d) The control system receives the position information and drives the robot to move in a direction to avoid collision.

\section{Conclusions}

Based on the refraction of electric field lines, a new flexible tactile sensor was designed. The proposed tactile sensor can detect the contact position in two directions in the same conductive layer. Position coordinate information was extracted based on the distribution function of potential gradient. A sensor model was simulated and analyzed, and sensors were successfully fabricated and tested. The experimental results showed that the sensor was suitable for position detection during contact. The experiments on the UR5 manipulator showed that the sensor exhibited good flexibility to cover the manipulator and that its accuracy in contact position detection can meet the requirements of robot collision avoidance. The sensor is not only simple in structure and inexpensive but also capable of providing a feasible solution for the safety of human-robot interaction.

\section{Acknowledgments}

This study is supported by the National Natural Science Foundation of China (Nos. 51175084 and 51575111). 


\section{References}

1 H. Ashrafian: Sci. Eng. Ethics 21 (2015) 317.

2 H. Wu and J. Yang: China Saf. Sci. J. 21 (2011) 79 (in Chinese).

3 K. Ikuta, H. Ishii, and M. Nokata: Int. J. Robot. Res. 22 (2003) 281.

4 V. J. Lumelsky and E. Cheung: IEEE T. Syst. Man. Cy-s. 23 (1993) 194.

5 N. Barnes and Z. Liu: Pattern. Recogn. 37 (2004) 299.

6 T. Xia, M. Yang, and R. Yang: Control and Decis. 25 (2010) 1 (in Chinese).

7 D. Vernon: Automatica 30 (1994) 731.

8 S. Ganapathy: Pattern Recogn. Lett. 2 (1984) 401.

9 M. Nokata, K. Ikuta, and H. Ishii: Proc. 2002 IEEE Int. Conf. Robotics and Automation (IEEE, 2002) 19911996.

10 F. Flacco, T. Kroger, A. D. Luca, and O. Khatib: Proc. 2012 IEEE Int. Conf. Robotics and Automation (IEEE, 2012) 338-345.

11 L. Seminara, L. Pinna, M. Valle, L. Basiricò, A. Loi, P. Cosseddu, A. Bonfiglio, A. Ascia, M. Biso, A. Ansaldo, D. Ricci, and G. Metta: IEEE Sens. J. 13 (2013) 4022.

12 G. H. Büscher, R. Kõiva, C. Schürmann, R. Haschke, and H. J. Ritter: Robot. Auton. Syst. 63 (2015) 244.

13 X. Zhu, H. Chen, L. Zhu, H. Wang, and W. Zhang: Opt. Eng. 53 (2014) 1.

14 Z. Pan and Z. Zhu: Ind. Robot. 32 (2005) 485.

15 A. Rana, J. P. Roberge, and V. Duchaine: IEEE Sens. J. 16 (2016) 7853.

16 V. Francesco, P. Fiorini, and K. Suzuki: Sensors 16 (2016) 1928.

17 Y. Zhang, G. Laput, and C. Harrison: Proc. 2017 CHI Conf. Human Factors in Computing Systems (ACM, 2017) 1-14.

18 H. Wu, J. Chen, Y. Su, Z. Li, and J. Ye: Sens. Actuators, A 242 (2016) 146.

19 V. A. Il'in, A. N. Mordvinov, and D. A. Petrov: J. Exp. Theor. Phys. 120 (2015) 161.

20 W. Liu, Y. Fu, X. Zou, P. Wang, and X. Wang: IEEE Trans. Plasma Sci. 42 (2014) 2574.

21 S. Wei, C. Shi, Z. Li, C. Tao, and C. Yan: J. Southwest China Normal University (Natural Science Edition) 38 (2013) 158 (in Chinese).

22 S. Gergo, J. Lewis, E. Vick, and D. Temple: J. Soc. Inf. Display 13 (2005) 575. 